\title{
Correlation between apparent diffusion coefficient and pathological characteristics of patients with invasive breast cancer
}

\author{
Yuhui Chen, Jiandong Wang, Xiuxiu Zhang, Wuyao Yang, Hongye Chen, Baoshi Bao, Yue Qiu, Lin Tian \\ Department of General Surgery, The First Medical Center, Chinese PLA General Hospital, Beijing, China \\ Contributions: (I) Conception and design: Y Chen, J Wang; (II) Administrative support: J Wang; (III) Provision of study materials or patients: J Wang, \\ W Yang, H Chen, Y Chen; (IV) Collection and assembly of data: Y Chen, X Zhang, B Bao, Y Qiu, L Tian; (V) Data analysis and interpretation: Y \\ Chen, J Wang; (VI) Manuscript writing: All authors; (VII) Final approval of manuscript: All authors. \\ Correspondence to: Jiandong Wang. Chief Physician, Master Supervisor, Department of General Surgery, The First Medical Center, Chinese PLA \\ General Hospital, Haidian, Beijing 100039, China. Email: wangjiandong@188.com.
}

Background: There is insufficient research on the correlation between the apparent diffusion coefficient and clinicopathological characteristics of breast cancer patients. The present study is to investigate the correlation between the apparent diffusion coefficient and pathological characteristics of patients with invasive breast cancer.

Methods: From January 2019 to September 2020, 122 cases of invasive breast cancer and 21 cases of benign tumors were retrospectively enrolled. The apparent diffusion coefficient was compared between the two groups, and the correlation between the apparent diffusion coefficient and the pathological characteristics of the patients with invasive breast cancer were analyzed.

Results: Compared with the benign tumor group, the apparent diffusion coefficient in the invasive breast cancer group was significantly lower $\left(0.89 \pm 0.17\right.$ vs. $\left.1.47 \pm 0.2710^{-3} \mathrm{~mm}^{2} / \mathrm{s}, \mathrm{P}=0.000\right)$. Using the apparent diffusion coefficient to diagnose patients with invasive breast cancer, the area under receiver operating characteristic (ROC) curve was $0.966 \pm 0.021$ [95\% confidence interval (CI): 0.924-1.000, P=0.000], and the best diagnostic cut-off value was $1.16\left(10^{-3} \mathrm{~mm}^{2} / \mathrm{s}\right)$, with sensitivity and specificity of 0.905 and 0.902 , respectively. The apparent diffusion coefficient was used to diagnose vascular tumor thrombus in patients with invasive breast cancer. The area under the ROC curve was $0.641 \pm 0.068$ (95\% CI: $0.508-0.774$, $\mathrm{P}=0.047)$, and the best diagnostic threshold was $0.835\left(10^{-3} \mathrm{~mm}^{2} / \mathrm{s}\right)$, with sensitivity and specificity of 0.676 and 0.650 , respectively. The apparent diffusion coefficient in patients with high expression of Ki-67 (\%) was significantly reduced $\left(0.87 \pm 0.17\right.$ vs. $\left.1.00 \pm 0.1610^{-3} \mathrm{~mm}^{2} / \mathrm{s}, \mathrm{P}=0.000\right)$. The apparent diffusion coefficient was not significantly correlated with age, menopause, lesion size, estrogen receptor, progesterone receptor, or lymph node metastasis in patients with invasive breast cancer $(\mathrm{P}>0.05)$.

Conclusions: In patients with invasive breast cancer the apparent diffusion coefficient was significantly reduced. It was able to differentiate invasive breast cancer and vascular tumor thrombus, and was also related to $\mathrm{Ki}-67$ (\%) high expression.

Keywords: Apparent diffusion coefficient; invasive breast cancer; Ki-67; pathological features; vascular tumor thrombus

Submitted Nov 11, 2020. Accepted for publication Dec 29, 2020.

doi: $10.21037 /$ atm-20-7746

View this article at: http://dx.doi.org/10.21037/atm-20-7746 


\section{Introduction}

The high incidence rate of breast cancer makes it one of the most common malignant tumors in women and, moreover, the incidence rate is still increasing (1-3). It is still one of the important causes of death in female patients (4-7). Routine examination methods include ultrasound examination, breast molybdenum target examination, breast MRI examination and biopsy, each with its own advantages and disadvantages. Breast MRI has the great advantage of preoperative evaluation of a range of lesions, especially the detection of small lesions (8-11). It has high value in qualitative diagnosis of breast cancer, but there are still many deficiencies in quantitative diagnosis. The apparent diffusion coefficient can quantitatively analyze the diffusion of water molecules in tissues and provide certain quantitative diagnostic information for breast cancer. At present, studies have confirmed that the apparent diffusion coefficient is significantly associated with clinical characteristics of breast cancer patients, but relevant research is still insufficient, and many studies do not differentiate breast cancer in situ and invasive cancer, so the clinical value is limited (12-14). Moreover, the correlation between the apparent diffusion coefficient and pathological characteristics of patients with invasive breast cancer is also insufficiently researched. Finally, in the present study, we also found that the apparent diffusion coefficient was related to the formation of vascular tumor thrombus, which is rarely reported in previous studies.

The aim of the present study was to investigate the correlation between apparent diffusion coefficient and pathological characteristics of patients with invasive breast cancer.

We present the following article in accordance with the STARD reporting checklist (available at http://dx.doi. org/10.21037/atm-20-7746).

\section{Methods}

\section{General data}

From January 2019 to September 2020, 122 cases of invasive breast cancer and 21 cases of benign breast tumor admitted to our hospital were retrospectively enrolled as the study group and control group, respectively. The inclusion criteria of invasive breast cancer group were: (I) invasive breast cancer; (II) newly diagnosed patients who had not received special treatment such as neoadjuvant therapy before operation; (III) 18-65 years old; (IV) complete data set available. The exclusion criteria were: (I) carcinoma in situ or benign tumor; (II) preoperative radiotherapy, chemotherapy or other special treatment; (III) patient did not undergo relevant MRI examination at Chinese PLA General Hospital; (IV) inflammatory breast cancer; (V) other malignant tumors; (VI) lack of relevant data.

For the control group, the inclusion criteria were: (I) benign tumor confirmed by pathology (e.g., fibroadenoma); (II) newly diagnosed patient who had not received special treatment before operation; (III) 18-65 years old; (IV) complete data set available. The exclusion criteria were: (I) carcinoma in situ or invasive carcinoma; (II) no breast enhancement MRI examination or lack of relevant parameters in our hospital; (III) inflammatory breast cancer; (IV) lack of relevant data. Individual consent for this retrospective analysis was waived. The ethical approval statement was not required due to the following reasons: (I) the study protocol was consistent with the Declaration of Helsinki (as revised in 2013); (II) our study was a retrospectively observational study, and we only studied the clinical data of the patients, which will not bring any harm to the patients; (III) we will try our best to protect the information provided by patients from disclosing personal privacy.

\section{Examination methods}

The patients in the invasive breast cancer group underwent dynamic enhanced MRI examination using a Siemens 3.0T MRI scanner with gadopentetate meglumine $(0.2 \mathrm{mmol} / \mathrm{kg})$ as the contrast agent administered intravenously in the forearm at a flow rate of $2.5 \mathrm{~mL} / \mathrm{s}$. Dynamic scanning was performed once, then the contrast agent was injected followed by a saline flush. Six consecutive acquisitions were performed before and after enhancement.

\section{Data collection}

(I) General data: age, onset site, smoking history, family history of alcoholism, menopause, complications. (II) MRI features: apparent diffusion coefficient, MRI early enhancement rate, dynamic enhanced curve. (III) Pathological features: lesion size, estrogen receptor (ER), progesterone receptor (PR), Ki-67 (\%), human epidermal growth factor receptor-2 (HER-2), axillary lymph node metastasis, supraclavicular lymph node metastasis, distant metastasis, invasion of skin or chest wall and pathological type. 
Table 1 Comparison of general data between the two groups

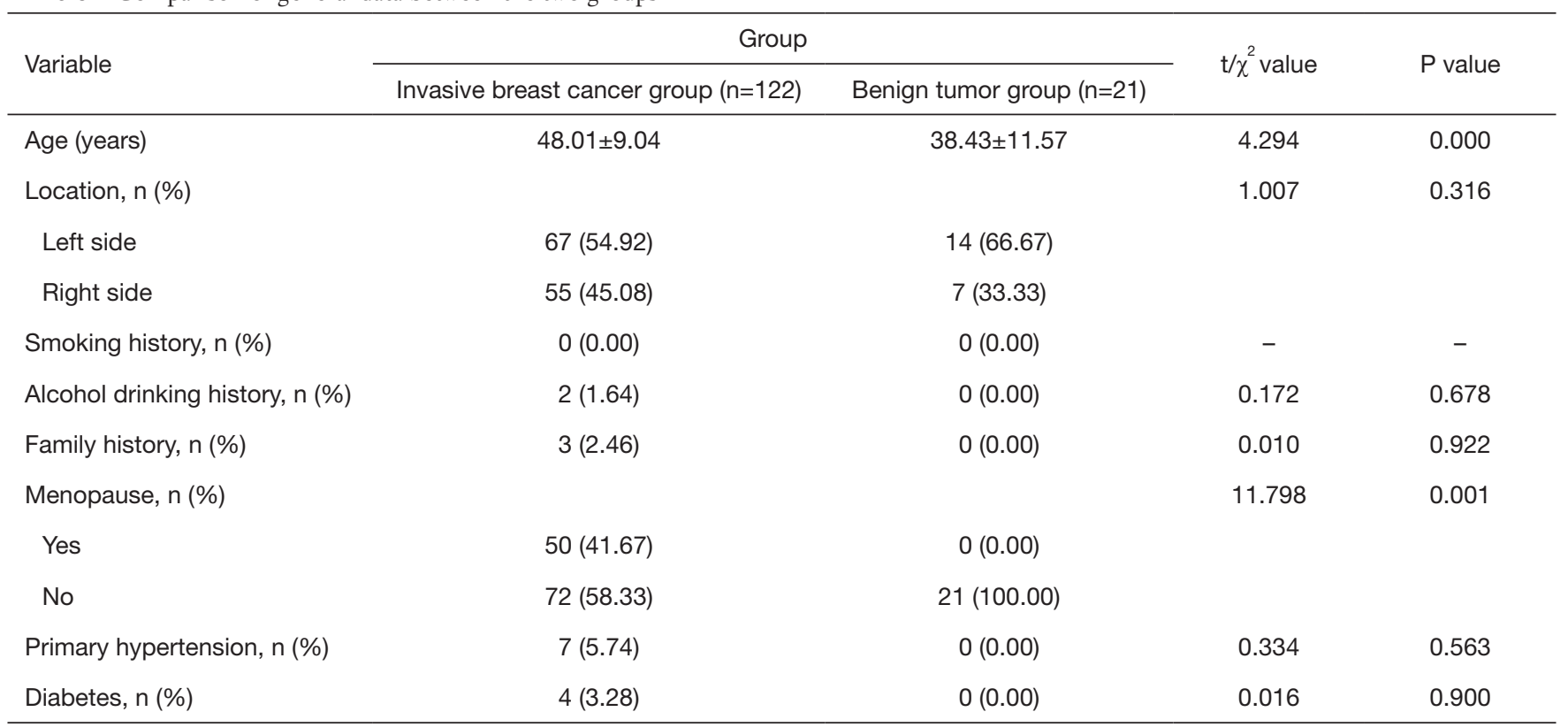

\section{Definitions}

ER, PR and Ki-67 are commonly used to guide the treatment of breast cancer. ER $\geq 10 \%$ was defined as high expression, otherwise defined as low expression; $\mathrm{PR} \geq 10 \%$ was defined as high expression, otherwise defined as low expression; Ki-67 (\%) >14\% was defined as high expression, otherwise defined as low expression.

\section{Quality control}

In our study, we found that the clinical data of some patients were incomplete. In order to exclude the deviation of results, we had excluded these patients. Moreover, we designed strict inclusion and exclusion criteria. In addition, we chose objective indicators to avoid recall bias. Finally, we chose two persons to input data independently. If the data was inconsistent, a third person would check it.

\section{Statistical analysis}

SPSS26.0 was used to complete the data analysis. When $\mathrm{P}<0.05$, the difference was statistically significant. The measurement data of the two groups was analyzed by $t$-test and expressed as mean \pm standard deviation; the count data were analyzed by chi square test and expressed as n (\%). Pearson's linear correlation was used to analyze the correlation between two measurement values, and receiver operating characteristic curve (ROC) was used to analyze the value of the apparent diffusion coefficient in the diagnosis of invasive breast cancer and vascular tumor thrombus.

\section{Results}

\section{General data of the two groups}

Compared with the benign tumor group, the age and menopause rate of the invasive breast cancer group were greater $(\mathrm{P}<0.05)$. There was no significant difference in the lesion location, smoking history, alcohol drinking history, family history and complications between the two groups $(\mathrm{P}>0.05)$ (Table 1).

\section{Size of lesions in the two groups}

There was no significant difference in the size of lesions between the two groups $(\mathrm{P}>0.05)$ (Table 2).

\section{MRI parameters of the two groups}

The apparent diffusion coefficient of the invasive breast cancer group was significantly lower than that of the benign tumor group $\left(0.89 \pm 0.17 v s .1 .47 \pm 0.2710^{-3} \mathrm{~mm}^{2} / \mathrm{s}, \mathrm{P}=0.000\right)$. 
Table 2 Comparison of lesion size between the two groups

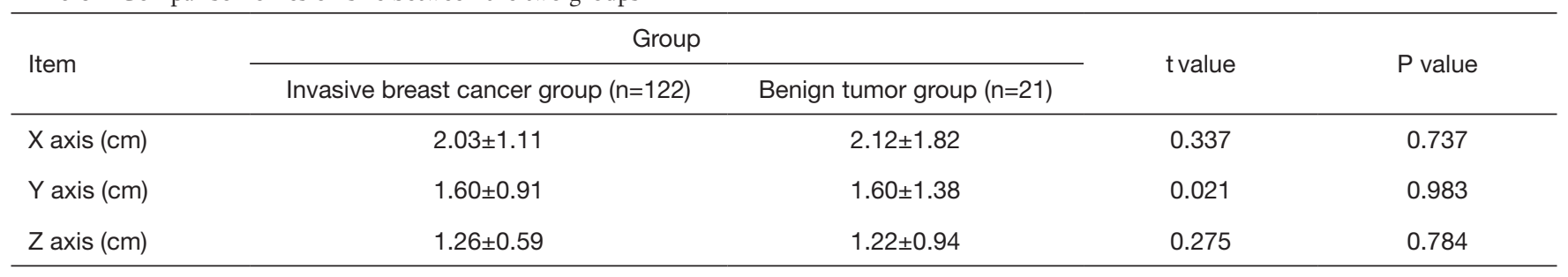

Table 3 Comparison of MRI parameters between the two groups

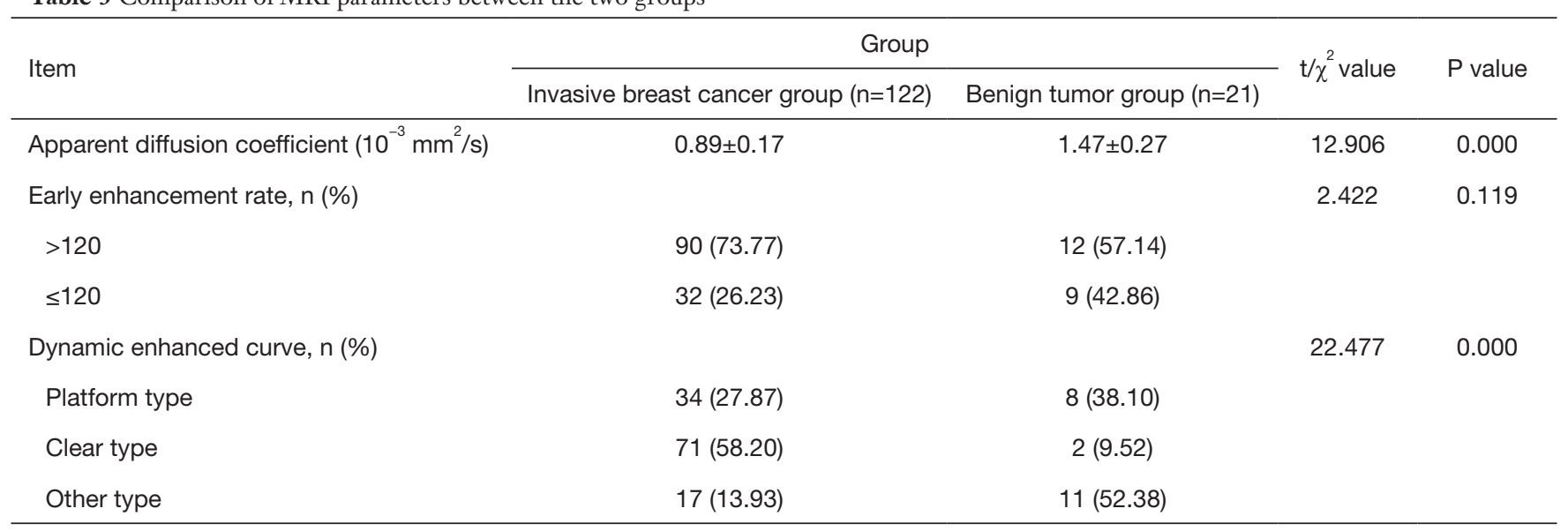

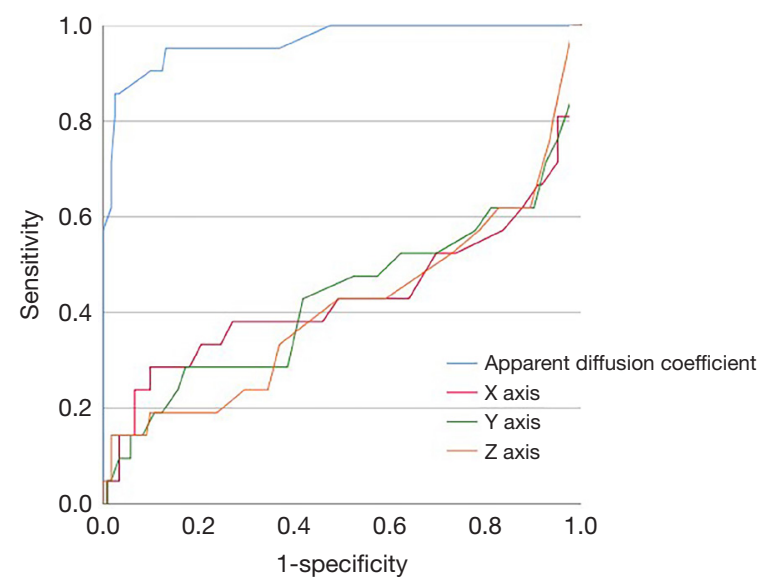

Figure 1 Diagnostic value of apparent diffusion coefficient of diffusion-weighted imaging in invasive breast cancer.

The dynamic enhanced curve of the invasive breast cancer group was significantly different from that of the benign tumor group, and the proportion of enhanced platform clearance type among the invasive breast cancer patients was increased $(\mathrm{P}<0.05)$. There was no significant difference in the early enhancement rate between the two groups
$(\mathrm{P}=0.567)$ (Table 3).

Value of apparent diffusion coefficient of diffusionweighted imaging in the diagnosis of invasive breast cancer

The area under the ROC curve was $0.966 \pm 0.021$ [95\% confidence interval $(\mathrm{CI}): 0.924-1.000, \mathrm{P}=0.000]$, and the best diagnostic cut-off was $1.16\left(10^{-3} \mathrm{~mm}^{2} / \mathrm{s}\right)$, with sensitivity and specificity of 0.905 and 0.902 , respectively (Figure 1, Table 4).

\section{Pathological characteristics of invasive breast cancer}

The pathological features of patients with invasive breast cancer are shown in Table 5.

\section{Correlation between apparent diffusion coefficient of diffusion-weighted imaging and pathological characteristics of patients with invasive breast cancer}

Pearson linear correlation analysis showed no significant difference between the apparent diffusion coefficient and lesion size $(\mathrm{P}>0.05)$ (Table 6). 
Table 4 Diagnostic value of apparent diffusion coefficient of diffusion-weighted imaging in invasive breast cancer

\begin{tabular}{lcccc}
\hline Variable & Area under curve & Standard deviation & P value & 95\% confidence interval \\
\hline Apparent diffusion coefficient & 0.966 & 0.021 & 0.000 & $0.924-1.000$ \\
X axis of lesions & 0.433 & 0.087 & 0.328 & $0.262-0.604$ \\
Y axis of lesions & 0.430 & 0.082 & 0.306 & $0.268-0.592$ \\
Z axis of lesions & 0.410 & 0.079 & 0.190 & $0.255-0.566$ \\
\hline
\end{tabular}

Table 5 Pathological characteristics of patients with invasive breast cancer

\begin{tabular}{cc}
\hline Group & Invasive breast \\
cancer $(n=122)(\%)$
\end{tabular}

Her-2

$(-)$

22 (18.03)

$(1+)$

$28(22.95)$

$(2+)$

$54(44.26)$

$(3+)$

18 (14.75)

Ki-67 (\%) high expression

$98(80.33)$

ER (\%) positive

$98(80.33)$

PR (\%) positive

$93(76.23)$

Axillary lymph node metastasis

40 (32.79)

Axillary lymph node metastasis

$1.39 \pm 3.19$

Subclavian lymph node metastasis

$0(0.00)$

Supraclavicular lymph node metastasis

$1(0.82)$

Invasion of skin or chest wall

$2(1.64)$

$0(0.00)$

Distant metastasis

Specific type

$111(90.98)$

Nonspecific type

$20(16.39)$

Vascular tumor thrombus

ER, estrogen receptor; PR, progesterone receptor; Her-2, human epidermal growth factor receptor-2; (-) means that negative staining results was observed or the staining intensity of cell membrane staining was only shown in $<10 \%$ of tumor cells; (1+) means some light and barely visible cell membrane staining, only partial staining was shown in more than $10 \%$ of tumor cells; (2+) means weak to moderate intact cell membrane staining, and the intensity was more than $10 \%$ and $<30 \%$ of tumor cells; 3 represents strong intact cell membrane staining, and the intensity is more than $30 \%$ of tumor cells.
Table 6 Correlation between apparent diffusion coefficient of diffusion-weighted imaging and lesion size

\begin{tabular}{lcc}
\hline Category & r value & $P$ value \\
\hline$X$ axis & 0.054 & 0.556 \\
$Y$ axis & 0.061 & 0.504 \\
$Z$ axis & 0.158 & 0.083 \\
\hline
\end{tabular}

\section{Association of apparent diffusion coefficient with}

pathological characteristics, age and menopause in patients with invasive breast cancer

The apparent diffusion coefficient of Ki-67 (\%) positive patients was significantly decreased $(0.87 \pm 0.17 v s .1 .00 \pm 0.16$ $\left.10^{-3} \mathrm{~mm}^{2} / \mathrm{s}, \mathrm{P}=0.000\right)$. There was no significant correlation between the apparent diffusion coefficient and HER-2, lymph node metastasis, pathological type, age or menopause in patients with invasive breast cancer $(\mathrm{P}>0.05)$ (Table 7).

\section{Value of apparent diffusion coefficient in the diagnosis of vascular tumor thrombus}

The area under ROC curve was $0.641 \pm 0.068$ (95\% CI: $0.508-0.774, \mathrm{P}=0.047)$, and the best diagnostic cut-off value was $0.83510^{-3} \mathrm{~mm}^{2} / \mathrm{s}$, with sensitivity and specificity of 0.676 and 0.650 , respectively (Figures 2,3 ).

\section{Value of apparent diffusion coefficient in the diagnosis of axillary lymph node metastasis}

The area under ROC curve was $0.528 \pm 0.055$ (95\% CI: 0.419-0.637, $\mathrm{P}=0.616$ ) (Figure 4). 
Table 7 Association of apparent diffusion coefficient with pathological characteristics, age and menopause in patients with invasive breast cancer

\begin{tabular}{|c|c|c|c|}
\hline Group & Apparent diffusion coefficient $\left(10^{-3} \mathrm{~mm}^{2} / \mathrm{s}\right)$ & t value & $P$ value \\
\hline$(-)$ or $(1+)(n=50)$ & $0.91 \pm 0.15$ & & \\
\hline$(2+)$ or $(3+)(n=72)$ & $0.88 \pm 0.19$ & & \\
\hline Axillary lymph node metastasis & & 0.315 & 0.753 \\
\hline No $(n=82)$ & $0.89 \pm 0.18$ & & \\
\hline Pathological type of breast cancer & & 1.556 & 0.122 \\
\hline Specific type $(n=11)$ & $0.96 \pm 0.19$ & & \\
\hline Nonspecific type $(n=111)$ & $0.88 \pm 0.17$ & & \\
\hline Low expression $(n=24)$ & $1.00 \pm 0.16$ & & \\
\hline ER positive & & 0.369 & 0.713 \\
\hline Yes $(n=98)$ & $0.89 \pm 0.16$ & & \\
\hline No $(n=24)$ & $0.90 \pm 0.22$ & & \\
\hline PR positive & & 0.682 & 0.497 \\
\hline Yes $(n=92)$ & $0.90 \pm 0.17$ & & \\
\hline No $(n=30)$ & $0.87 \pm 0.17$ & & \\
\hline Vascular tumor thrombus & & 0.682 & 0.497 \\
\hline Menopause & & 1.287 & 0.201 \\
\hline Yes $(n=50)$ & $0.91 \pm 0.15$ & & \\
\hline No $(n=72)$ & $0.87 \pm 0.19$ & & \\
\hline
\end{tabular}

ER, estrogen receptor; PR, progesterone receptor; Her-2, human epidermal growth factor receptor-2; (-) means that negative staining results was observed or the staining intensity of cell membrane staining was only shown in less than $10 \%$ of tumor cells; (1+) means some light and barely visible cell membrane staining, only partial staining was shown in more than $10 \%$ of tumor cells; (2+) means weak to moderate intact cell membrane staining, and the intensity was more than $10 \%$ and less than $30 \%$ of tumor cells; (3+) represents strong intact cell membrane staining, and the intensity is more than $30 \%$ of tumor cells. 


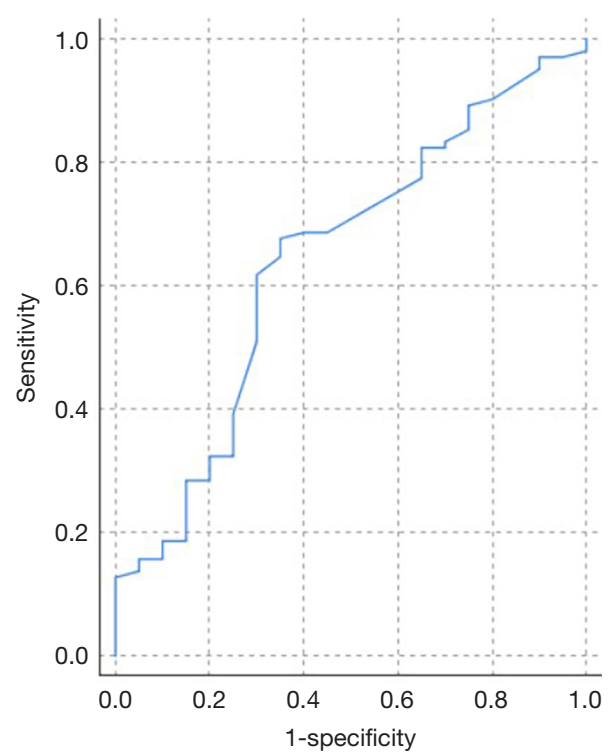

Figure 2 Value of apparent diffusion coefficient in the diagnosis of vascular tumor thrombus.

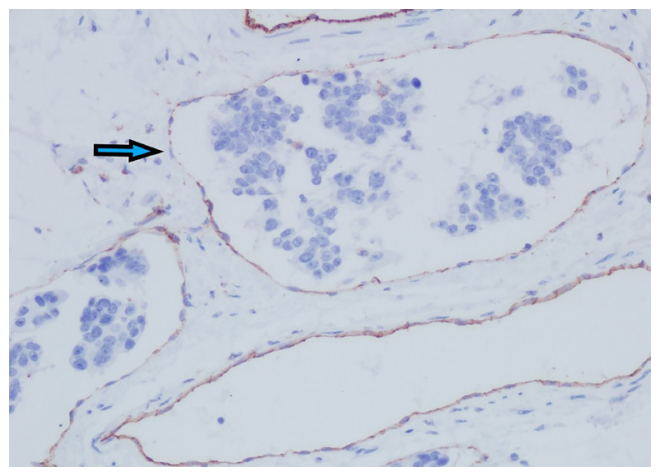

Figure 3 Vascular tumor thrombus in breast cancer tissue (immunohistochemical staining; arrow showed that the formation of vascular tumor thrombus; $\times 20$ ).

\section{Correlation between apparent diffusion coefficient of diffusion-weighted imaging and molecular subtype in invasive breast cancer}

There was no correlation between apparent diffusion coefficient and luminal subtypes $(\mathrm{P}=0.525)$ (Figure 5).

\section{Adverse events}

No adverse reactions occurred during MRI examination.

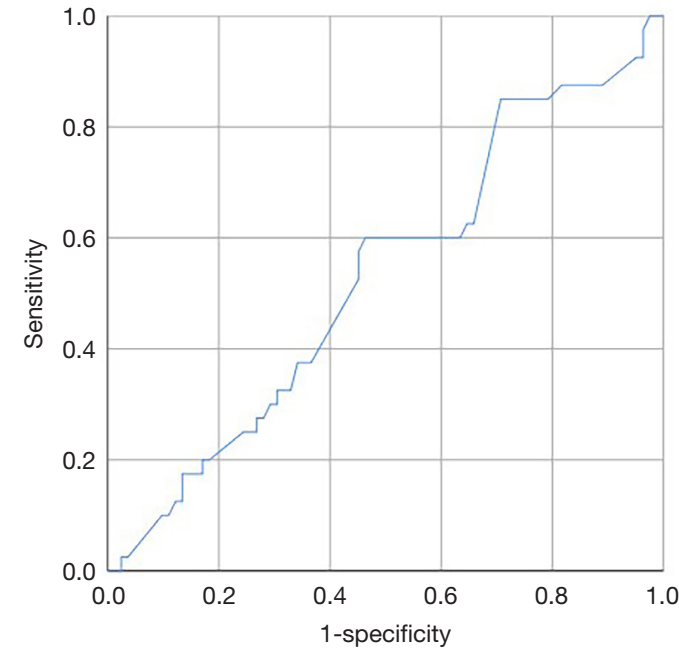

Figure 4 Value of apparent diffusion coefficient in the diagnosis of axillary lymph node metastasis.

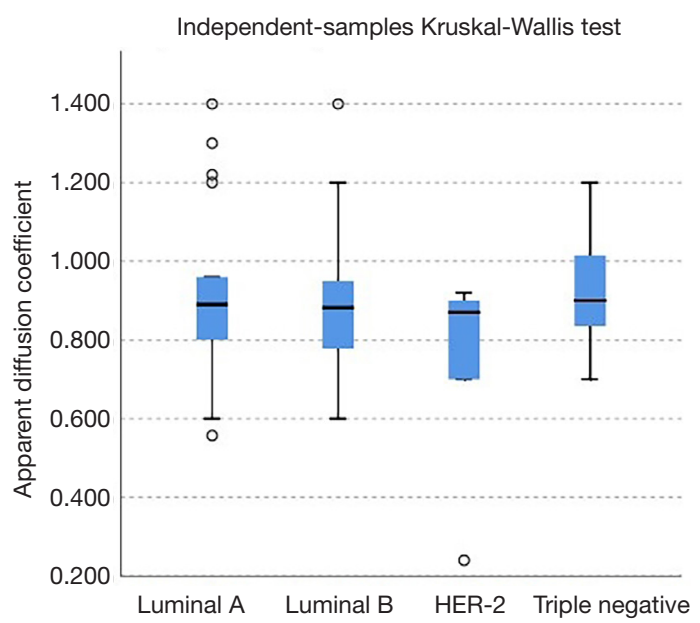

Figure 5 Correlation between apparent diffusion coefficient of diffusion-weighted imaging and luminal subtypes in invasive breast cancer.

\section{Discussion}

This study explored the correlation between the apparent diffusion coefficient and the pathological characteristics of patients with invasive breast cancer. The results showed that the apparent diffusion coefficient of patients with invasive breast cancer was significantly lower, and it had a good value for differentiating invasive breast cancer from benign tumor as well as identifying vascular tumor 
thrombus. Moreover, it was related to positive expression of $\mathrm{Ki}-67$.

The apparent diffusion coefficient is the parameter that describes the diffusion velocity and range of different water molecules in diffusion-weighted MRI. It has high application value in a variety of tumors. Although it is a common parameter in the MRI examination of breast cancer patients, it is often ignored by clinicians due to their lack of understanding of the apparent diffusion coefficient. In patients with breast cancer, the apparent diffusion coefficient is mainly used to analyze the movement of water molecules in tissue cells. Patients with a malignant tumor have higher cell density and less tissue space, leading to limited free diffusion of water molecules, which manifests as a decrease in the apparent diffusion coefficient. This is the theoretical basis of using the apparent diffusion coefficient to diagnose invasive breast cancer, and our study results showed that the apparent diffusion coefficient of patients with invasive breast cancer was significantly lower than that of patients with benign tumors.

Researchers are beginning to pay attention to the role of the apparent diffusion coefficient in the evaluation of breast cancer patients. Studies have shown that the apparent diffusion coefficient can be used as a potential biomarker of complete remission after neoadjuvant chemotherapy for breast cancer (15). If neoadjuvant chemotherapy is effective, most of the cancer cells will be killed, and the apparent diffusion coefficient will increase. Other studies have shown that the apparent diffusion coefficient had good value in the diagnosis of invasive ductal carcinoma and breast ductal carcinoma in situ, with the apparent diffusion coefficient of invasive ductal carcinoma being lower (16). These studies support our study results, indicating that the apparent diffusion coefficient can be used for the preliminary diagnosis of invasive breast cancer. In our study, the area under ROC curve was $0.987 \pm 0.012$ (95\% CI: $0.965-1.000, \mathrm{P}=0.000$ ), and the best diagnostic cut-off was $1.25\left(10^{-3} \mathrm{~mm}^{2} / \mathrm{s}\right)$, with sensitivity and specificity of 0.909 and 0.987 , respectively. We suggest that breast cancer patients with an apparent diffusion coefficient less than $1.25\left(10^{-3} \mathrm{~mm}^{2} / \mathrm{s}\right)$ should be aware of the possibility of an invasive tumor.

Furthermore, we showed that the apparent diffusion coefficient of invasive breast cancer patients correlated with vascular tumor thrombus, making it also valuable for the diagnosis of this condition. The lower the apparent diffusion coefficient, the higher the Ki-67 expression rate, and the greater the possibility of vascular tumor thrombus.
Some studies have shown that the apparent diffusion coefficient is related to $\mathrm{PR}$ positivity and axillary lymph node metastasis in breast cancer patients $(13,14)$. The results of our study showed that the apparent diffusion coefficient was not related to PR positivity and axillary lymph nodes in patients with invasive breast cancer, but significantly correlated with Ki-67. The reason for the inconsistent results may be that the number of cases included in the previous study was limited at only 83 patients whereas we included 122 patients in our study. Moreover, the apparent diffusion coefficient has been associated with nuclear grade, histological grade, lymph node positive, ER negative, PR negative, HER-2 negative and $\mathrm{Ki}-67$ positive expression (17). In addition, another study showed that the apparent diffusion coefficient can be used to evaluate changes in $\mathrm{Ki}-67$ expression after neoadjuvant chemotherapy in breast cancer (18). Ki-67 is an antigen associated with cell proliferation and generally less than $10 \%$ with benign tumors, but more than $10 \%$ with malignant tumors. In patients with invasive breast cancer, the higher Ki-67 expression, the faster the proliferation of cancer cells, and the greater the likelihood of metastasis (19-22). As the Ki-67 level increases, cell growth increases, so the tumor cell density is higher, resulting in a decrease of the apparent diffusion coefficient. Recent studies showed that $\mathrm{Ki}-67$ can used as a good predictor in the diagnosis of lymph node metastasis, recurrence and death (23-26).

In addition, the formation of vascular tumor thrombus is a risk factor for poor prognosis of patients with invasive breast cancer. When the apparent diffusion coefficient is reduced, the density of tumor cells is high, and thus tumor cells are more likely to enter the vessels, which will lead to lymph node metastasis or distant metastasis. This study showed that the apparent diffusion coefficient had a certain value in the diagnosis of vascular tumor thrombus, and there are few related studies. However, the apparent diffusion coefficient was not found to be related to lymph node metastasis or distant metastasis in this study, which may be caused by the limited cases enrolled in this study. In summary, a decreased apparent diffusion coefficient may be a risk factor for poor prognosis in patients with invasive breast cancer.

In conclusion, the apparent diffusion coefficient was significantly lower in patients with invasive breast cancer, which showed good value in differentiating invasive breast cancer and vascular tumor thrombus. Moreover, it was related to high expression of $\mathrm{Ki}-67$ (\%) in patients with invasive breast cancer. 


\section{Limitations}

This was a retrospectively study. The characteristics of the distribution of apparent diffusion coefficient values in MRI diffusion weighted imaging of surrounding glands were not available. Moreover, only patients admitted from January 2019 to September 2020 were enrolled in our study, therefore the follow-up time was too short. Therefore, we failed to study the correlation between the apparent diffusion coefficient of MRI and prognosis in our study. Finally, we failed to study the value of evaluating different apparent diffusion coefficient indicators in the predicting of status and proliferation rate of invasive breast cancer.

\section{Acknowledgments}

Funding: This study is supported by China Medical Education Association [2016] 001.

\section{Footnote}

Reporting Checklist: The authors have completed the STARD reporting checklist. Available at http://dx.doi. org/10.21037/atm-20-7746

Data Sharing Statement: Available at http://dx.doi. org/10.21037/atm-20-7746

Conflicts of Interest: All authors have completed the ICMJE uniform disclosure form (available at http://dx.doi. org/10.21037/atm-20-7746). The authors have no conflicts of interest to declare.

Ethical Statement: The authors are accountable for all aspects of the work in ensuring that questions related to the accuracy or integrity of any part of the work are appropriately investigated and resolved. Individual consent for this retrospective analysis was waived. The ethical approval statement was not required due to the following reasons: (I) the study protocol was consistent with the Declaration of Helsinki (as revised in 2013); (II) our study was a retrospectively observational study, and we only studied the clinical data of the patients, which will not bring any harm to the patients; (III) we will try our best to protect the information provided by patients from disclosing personal privacy.

Open Access Statement: This is an Open Access article distributed in accordance with the Creative Commons Attribution-NonCommercial-NoDerivs 4.0 International License (CC BY-NC-ND 4.0), which permits the noncommercial replication and distribution of the article with the strict proviso that no changes or edits are made and the original work is properly cited (including links to both the formal publication through the relevant DOI and the license). See: https://creativecommons.org/licenses/by-nc-nd/4.0/.

\section{References}

1. Oliver J, Quezada Urban R, Franco Cortés CA, et al. Latin American Study of Hereditary Breast and Ovarian Cancer LACAM: A Genomic Epidemiology Approach. Front Oncol 2019;9:1429.

2. Prysyazhnyuk AY, Bazyka DA, Romanenko AY, et al. Epidemiology of breast cancer in Ukraine with consideration of the factors of the Chornobyl accident. Probl Radiac Med Radiobiol 2019;24:150-68.

3. Adejumo AA, Ajamu OJ, Akanbi OO, et al. Epidemiology and Challenges of Managing Breast Cancer in Keffi, North-Central Nigeria: A Preliminary Report. Niger Med J 2019;60:193-7.

4. Koczkodaj P, Sulkowska U, Gotlib J, et al. Breast cancer mortality trends in Europe among women in perimenopausal and postmenopausal age (45+). Arch Med Sci 2019;16:146-56.

5. Chuang SC, Lin CH, Lu YS, et al. Mortality of Pregnancy Following Breast Cancer Diagnoses in Taiwanese Women. Oncologist 2020;25:e252-58.

6. Hanis TM, Yaacob NM, Hairon SM, et al. Modelling excess mortality among breast cancer patients in the North East Region of Peninsular Malaysia, 20072011: a population-based study. BMC Public Health 2019;19:1754.

7. Oliveira NPD, Santos Siqueira CAD, Lima KYN, et al. Association of cervical and breast cancer mortality with socioeconomic indicators and availability of health services. Cancer Epidemiol 2020;64:101660.

8. Debruhl ND, Lee SJ, Mahoney MC, et al. MRI Evaluation of the Contralateral Breast in Women with Recently Diagnosed Breast Cancer: 2-Year Follow-up. J Breast Imaging 2020;2:50-5.

9. Mouawad M, Biernaski H, Brackstone M, et al. DCE-MRI assessment of response to neoadjuvant SABR in early stage breast cancer: Comparisons of single versus three fraction schemes and two different imaging time delays post-SABR. Clin Transl Radiat Oncol 2019;21:25-31. 
10. Hu H, Wang Y, Zhang T, et al. Association of LncRNAGACAT3 with MRI features of breast cancer and its molecular mechanism. J BUON 2019;24:2377-84.

11. Cao K, Zhao B, Li XT, et al. Texture Analysis of Dynamic Contrast-Enhanced MRI in Evaluating Pathologic Complete Response (pCR) of Mass-Like Breast Cancer after Neoadjuvant Therapy. J Oncol 2019;2019:4731532.

12. Choi BB. Associations Between Apparent Diffusion Coefficient Values and the Prognostic Factors of Breast Cancer. J Comput Assist Tomogr 2019;43:931-6.

13. Surov A, Chang YW, Li L, et al. Apparent diffusion coefficient cannot predict molecular subtype and lymph node metastases in invasive breast cancer: a multicenter analysis. BMC Cancer 2019;19:1043.

14. Tezcan Ş, Uslu N, Ozturk FU, et al. Diffusion-Weighted Imaging of Breast Cancer: Correlation of the Apparent Diffusion Coefficient Value with Pathologic Prognostic Factors. Eur J Breast Health 2019;15:262-7.

15. Graña-López L, Herranz M, Macineira FA, et al. Apparent diffusion coefficient: Potential biomarker for complete response after neo-adjuvant chemotherapy in breast cancer. Breast J 2020;26:306-8.

16. Zhao S, Shao G, Chen P, et al. Diagnostic performance of minimum apparent diffusion coefficient value in differentiating the invasive breast cancer and ductal carcinoma in situ. J Cancer Res Ther 2019;15:871-5.

17. Ren C, Zou Y, Zhang X, et al. Diagnostic value of diffusion-weighted imaging-derived apparent diffusion coefficient and its association with histological prognostic factors in breast cancer. Oncol Lett 2019;18:3295-303.

18. Luo N, Ji Y, Huang X, et al. Changes in Apparent Diffusion Coefficient as Surrogate Marker for Changes in Ki-67 Index Due to Neoadjuvant Chemotherapy in Patients with Invasive Breast Cancer. Acad Radiol

Cite this article as: Chen Y, Wang J, Zhang X, Yang W, Chen H, Bao B, Qiu Y, Tian L. Correlation between apparent diffusion coefficient and pathological characteristics of patients with invasive breast cancer. Ann Transl Med 2021;9(2):143. doi: 10.21037/atm-20-7746
2019;26:1352-7.

19. Kang YJ, Lee HB, Kim YG, et al. Ki-67 Expression is a Significant Prognostic Factor Only When Progesterone Receptor Expression is Low in Estrogen Receptor-Positive and HER2-Negative Early Breast Cancer. J Oncol 2019;2019:7386734.

20. Fan M, Yuan W, Zhao W, et al. Joint Prediction of Breast Cancer Histological Grade and Ki-67 Expression Level Based on DCE-MRI and DWI Radiomics. IEEE J Biomed Health Inform 2020;24:1632-42.

21. Noske A, Anders SI, Ettl J, et al. Risk stratification in luminal-type breast cancer: Comparison of Ki-67 with EndoPredict test results. Breast 2020;49:101-7.

22. Rossi L, Verrico M, Tomao S, et al. Expression of ER, PgR, HER-2, and Ki-67 in core biopsies and in definitive histological specimens in patients with locally advanced breast cancer treated with neoadjuvant chemotherapy. Cancer Chemother Pharmacol 2020;85:105-11.

23. Chen C, Lu X. The expression of KI-67 and LEF-1 in patients after breast cancer resection and its effects on patients' prognosis. J BUON 2020;25:627-33.

24. Sood N. Ki-67, an elusive marker in the prognosis of breast cancer. Indian J Med Res 2019;150:214-6.

25. Kamranzadeh H, Ardekani RM, Kasaeian A, et al. Association between $\mathrm{Ki}-67$ expression and clinicopathological features in prognosis of breast cancer: A retrospective cohort study. J Res Med Sci 2019;24:30.

26. Arima N, Nishimura R, Osako T, et al. Ki-67 index value and progesterone receptor status can predict prognosis and suitable treatment in node-negative breast cancer patients with estrogen receptor-positive and HER2-negative tumors. Oncol Lett 2019;17:616-22.

(English Language Editor: K. Brown) 